\title{
Identification of glaucomatous visual field defects from examination of monocular photographs of the optic disc
}

\author{
R. A. HITCHINGS AND S. ANDERTON \\ From the Glaucoma Unit, Moorfields Eye Hospital, High Holborn, London WC1V 7 AN
}

\begin{abstract}
SUMMARY A study was carried out of monocular disc photographs from 33 eyes for which the visual fields on both static profile and kinetic perimetry has been performed. Physical signs looked for at the optic disc included thinning of the neuroretinal rim, angulation of retinal vessels, extension of laminar dots, undercutting of the neuroretinal rim, and absence and pallor of the neuroretinal rim. These signs together proved more accurate than kinetic Goldmann perimetry in identifying the presence of glaucomatous visual field defect. Of these signs angulation of the retinal vessels was the one most consistently present.
\end{abstract}

The examination of photographs has definite advantages over direct ophthalmoscopy. These are a stable image, the ability to superimpose a grid over the photographs and allow measurements to be made, and an image to retain for comparison with future images of the optic disc. By using photographs with a stereo effect a high degree of accuracy in the identification of visual field defects shown on kinetic perimetry may be obtained. ${ }^{1}$ By identifying arbitrary rim thickness to correspond with the presence of visual field defects seen with kinetic perimetry the use of a superimposed grid allows paramedical personnel also correctly to identify defects in the field of vision. ${ }^{2}$ Monocular photographs may well prove to be a less accurate method of identifying visual field defects. By relying on centrifugal extension of central pallor Shiose $^{3}$ could correctly identify many eyes with glaucomatous field defects. The promise shown by this pilot study was not borne out on mass screening. ${ }^{4}$ Because this author used only one sign suggesting a change in the appearance of the optic disc, it seemed worthwhile to see whether other signs or a combination of signs could increase the accuracy in identification of glaucomatous visual field defects from examination of the monocular photograph of the optic disc.

Sharma and Hitchings ${ }^{5}$ found a high degree of sensitivity and specificity when relying on a number of signs (angulation undercutting, laminar dots, pallor)

Correspondence to Mr R. A. Hitchings. to correlate with visual field defects seen with the Armaly/Drance technique. However, as similar signs were present in optic discs without visual field defects the question of early visual field defect was raised. In a recent study ${ }^{6}$ static profile perimetry identified absolute (1000 abs) defects not seen on Goldmann kinetic/static perimetry carried out by the Armaly/ Drance technique. ${ }^{7}$ It seemed worthwhile to re-examine the optic disc photographs for the patient examined in this comparative visual field study to see whether the same constellation of signs was of use in identifying visual field defects seen on static profile perimetry.

\section{Material and methods}

Optic disc photographs were available for comparison with visual field defects identified on static profile perimetry in a series of patients previously reported on. ${ }^{6}$ The photographs were taken by a standard Zeiss fundus camera and were of varying quality. Photographs of 33 eyes of the patients reported upon in this earlier series were available, and these form the basis of this report.

The photographs were examined for signs suggesting glaucomatous visual field defects. The presence of one or more of the signs listed below was considered suggestive. According to the location of the signs on the upper and lower half of the optic disc the visual field defect was placed in the corresponding lower and upper half of the visual field. These 


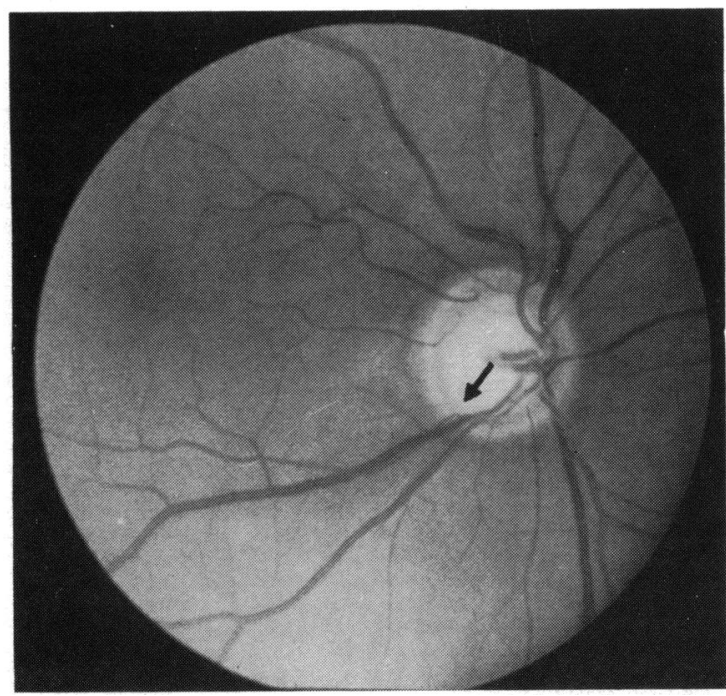

Fig. 1 Optic disc with thinning of the neuroretinal rim at the inferior pole (arrow).

predictions were then compared with the results obtained by perimetry by the Armaly/Drance technique and also with the results obtained by static profile perimetry.

Secondly, the frequency of the suggestive signs for any individual disc was noted.

Those features numbered suggestive of a glaucomatous visual field defect included:

(1) Any segment of neuroretinal rim less than one division of the optic grid previously described ${ }^{2}$ (Fig. 1). It should be pointed out that for this study only monocular photographs were available, and a clear

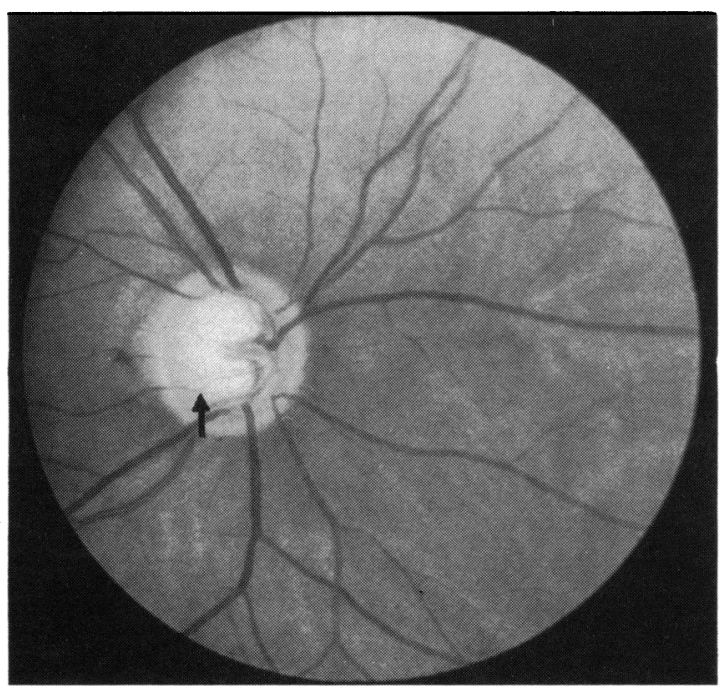

Fig. 2 'Angulation' (arrow) of a small blood vessel on the side wall of the optic cup.

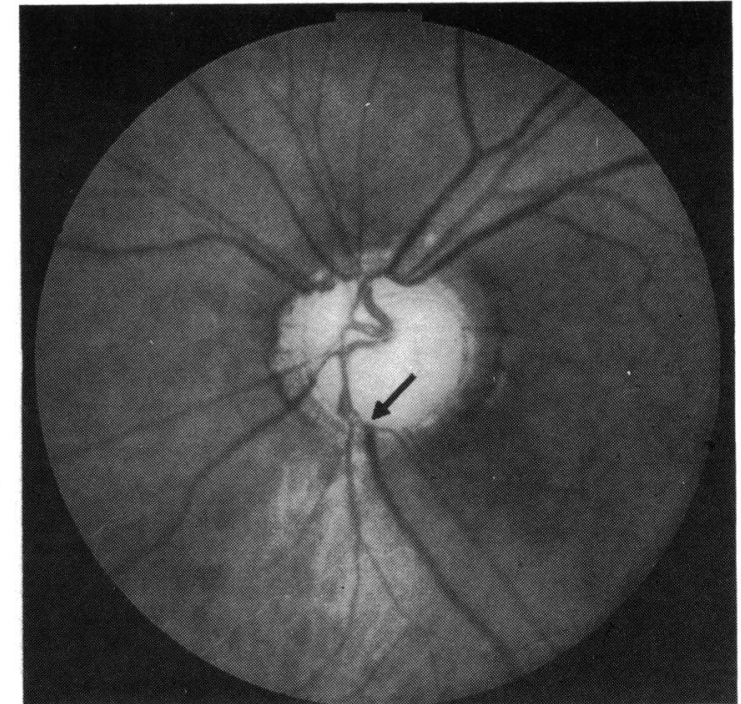

Fig. 3 'Undercutting' of the neuroretinal rim: shown by disappearance of retinal blood vessels beneath neuroretinal rim (arrow).

demarcation between rim and optic cup was not present in every case.

(2) 'Angulation' of a retinal vessel as it crossed the surface of the optic disc. 'Angulation' was considered to be present if a retinal vessel, of whatever size, did not follow a smooth curve as it crossed over the disc surface but showed a sudden change in direction resulting in a kink in the course of the vessel (Fig. 2) It was recognised that such angulation could result from a change in the vessel course along a horizontal or an anteroposterior axis or a combination of both.

(3) 'Undercutting' of the neuroretinal rim. The sign was considered to be present when a retinal blood vessel temporarily disappeared from view behind the neuroretinal rim (Fig. 3). Although this sign was typically seen in 'bean pot' cups, ${ }^{8}$ it could also be seen in one part of an optic disc which had a quite small optic cup.

(4) Absence of part of the neuroretinal rim (Fig. 4).

(5) Centrifugal extension of laminar dots (Fig. 5). Pallor was considered to be present if, typically, one part of the neuroretinal rim was demonstrably paler than the remainder, of if gross pallor existed throughout the neuroretinal rim.

\section{Results}

The optic disc photographs from 33 eyes were available for study.

The comparative rates of identification for the 2 types of visual field test and examination of the monocular photographs are set out in Table 1. 


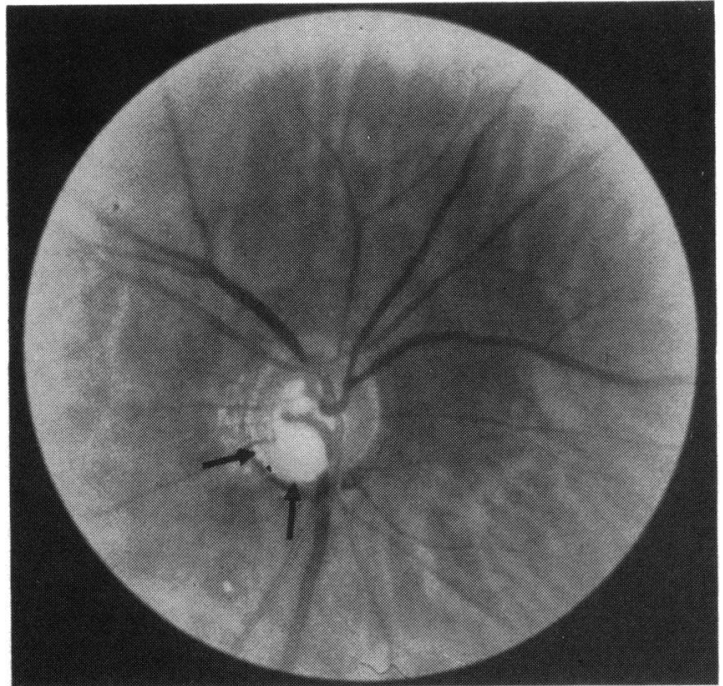

Fig. 4 'Absence' of neuroretinal rim (arrows).

Following the identification of eyes with a visual field defect on static profile perimetry those features previously noted which were present in the corresponding (opposite) half of the optic disc were identified. These are set out in Table 2. Angulation of retinal vessels was the most accurate in predicting the presence of a visual field defect. Those features

Table 1 Comparison of examination of monocular disc photograph with kinetic Goldmann perimetry in the identification of glaucomatous visual field defects

\begin{tabular}{|c|c|c|c|}
\hline Static perimetry & No. & $\begin{array}{l}\text { Correctly } \\
\text { identified by } \\
\text { kinetic } \\
\text { perimetry }\end{array}$ & $\begin{array}{l}\text { Correctly } \\
\text { identified by } \\
\text { examination of } \\
\text { monocular } \\
\text { photographs }\end{array}$ \\
\hline Full field & 1 & 1 & 0 \\
\hline Normal 'hemi' field & 14 & 14 & 11 \\
\hline $\begin{array}{l}\text { Upper and lower field } \\
\text { defects }\end{array}$ & 18 & 4 & 17 \\
\hline Total & 33 & 19 & 28 \\
\hline
\end{tabular}

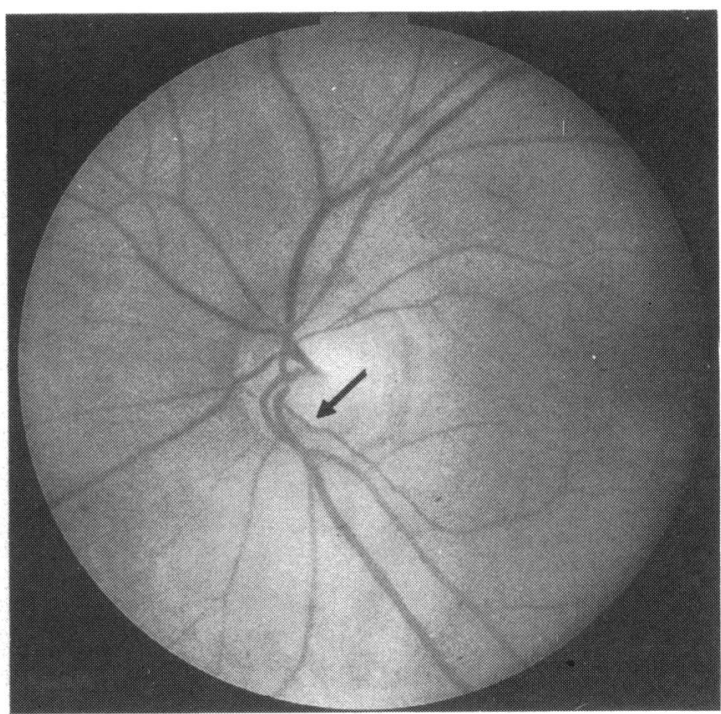

Fig. 5 Centrifugal extension of laminar dots (arrow).

present in the absence of angulation are set out in Table 3. It should be noted that pallor alone was the feature noted together with a visual field defect in 6 out of the 14 cases when angulation was not seen.

\section{Discussion}

This paper has used a number of physical signs visible on monocular photographs of the optic disc to predict the presence or absence of a visual field defect present on static profile perimetry. It has shown that summation of these physical signs-essentially what took place in the first part of the study-proved a more accurate indicator of the presence of a visual field defect than 3 isoptre perimetry by the Armaly/ Drance technique. For the patients in this study this type of perimetry was not particularly accurate, failing to identify (mainly absolute, $1000 \mathrm{asb}$ ) defects in 14 of the 33 eyes tested. Profile perimetry did not fail to identify a visual field defect noted with the Armaly/Drance technique.

Table 2 Presence of physical signs on the optic disc when a visual field defect exists in the appropriate (upper and lower) half of the visual field: 33 eyes, 53 hemivisual fields with a defect on static profile perimetry

\begin{tabular}{llcccc}
\hline Total no of visual field defects & \multicolumn{3}{l}{ Positive identification of a physical sign to correspond with a visual field defect } \\
\cline { 2 - 5 } & Thin rim & Angulation & Undercut rim & Laminar dots & Absence \\
\hline 53 & 28 & 39 & 19 & 21 & Pallor \\
\hline
\end{tabular}

Table 3 Physical signs present in the 14 hemifields where angulation was not recognised

\begin{tabular}{lccccc}
\hline Total & Thin rim & Angulation & Undercut rim & Laminar dot & Absence \\
\hline 14 & 5 & 0 & 2 & 3 & 5 \\
\hline
\end{tabular}


The second part of this study showed that, after analysis of the various physical signs present with a visual field defect, angulation of the retinal vessels was the sign most often present with visual field loss. It should be noted, however, that pallor alone was present in 6 of the 14 cases where angulation was not observed, and it is possible that in these cases optic atrophy without gross enlargement of the optic cup had taken place. ${ }^{9}$ Because of the frequency with which angulation of blood vessels corresponded with visual field loss it is worthwhile looking at this physical sign in more detail.

The concept of angulation follows 2 premises. The first is that the normally developed optic nerve head has retinal vessels on its surface which would follow a smooth curve when changing direction, for anything else might impede blood flow. The second premise is that the expanding optic cup in glaucoma rarely leaves retinal blood vessels on the disc surface unaffected, with the exception of overpassed vessels, ${ }^{8}$ but is followed by or associated with a change in the course of the retinal vessels as they remain in contact with the surface of the expanding optic cup. This change in direction terminates suddenly as the vessel continues along its original course over an unaffected part of the optic disc (Fig. 2). It was noted for the patients in this study that both small and large vessels may be affected by this process, and it may well be that small vessels could be affected at an earlier stage.

Sudden change in direction of retinal blood vessels on the surface of the optic disc has been commented upon before. Read and Spaeth ${ }^{10}$ described 'bayoneting' of blood vessels, noting that the affected blood vessel 'must bend sharply right at the edge of the disc so that there is no pink glial tissue between the blood vessel and disc edge'.

They note that bayoneting only indicates a sharpened rim which might otherwise not be apparent unless stereo viewing methods were used'. A sharp change in direction (angulation) need not be restricted to vessels at the optic disc edge but can be seen as a change in direction anywhere in the optic cup and is frequently seen well away from the disc edge (Fig. 2).

Herschler and Osler suggested ${ }^{11}$ that 'baring of the circumlinear vessel' was a sign of enlargement of the optic cup in ocular hypertensive patients and considered it to be present before the visual field loss occurs. According to these authors a circumlinear vessel is either a vein or arteriole which can serve as a marker to the border of the physiological cup. They felt that such a vessel was 'left behind when the optic cup enlarged,' becoming separated from the rim. Baring of this vessel was considered to be present when an area of pale tissue existed between the vessel and the neuroretinal rim. Examination of the photographs depicted in their article showed vessel angulation frequently to coexist with the baring of the circumlinear vessel, suggesting that both signs might indicate enlargement of the optic cup.

According to a later article ${ }^{12}$ (Goldmann) perimetry was performed on their patients. If this was always the case, then a number of their ocular hypertensive patients may well have had visual field defects present on static profile perimetry, as was noted for the patients in this series.

\section{CONCLUSION}

Monocular photographs of the optic disc may often provide sufficient visual clues to allow the identification of visual field defects present on static profile perimetry. It should be remembered, however, that, although the false negative rates for the prediction of visual field defect in the hemivisual field from examination of monocular photographs was low, the study design gave no opportunity to identify the false positive rate-the suggestion that a visual field defect existed where there were none. Such identification requires a further investigation, which is at present in progress. However, from the point of view of glaucoma screening the side on which to err is the false positive rather than the false negative, so that visual field defects, if present, are not missed.

Our thanks are due to Kay Mills for typing the manuscript.

\section{References}

1 Hitchings RA, Spaeth GL. The optic disc in glaucoma. II. Correlation of the appearance of the optic disc with the visual field. Br J Ophthalmol 1977; 61: 107-13.

2 Hitchings RA, Brown DB, Anderton SA. Glaucoma screening by means of an optic disc grid. Br J Ophthalmol 1983; 67: 352-5.

3 Shiose Y. 'Quantitative disc pattern' or a new parameter for glaucoma screening. Glaucoma 1979; 1: 41-9.

4 Shiose Y, Kommo K, Itoko T, Amano M, Kawase Y. New system for mass screening as part of automated multiphasic health screening process. Jpn J Clin Ophthalmol 1981; 25: 160-77.

5 Sharma N, Hitchings RA. The use of monocular disc photographs in the identification of glaucomatous visual field defect. $\mathrm{Br} \mathrm{J}$ Ophthalmol in press.

6 Hitchings RA, Anderton SA. A comparative study of visual field defects seen in patients with low-tension glaucoma and chronic simple glaucoma. Br J Ophthalmol 1983; 67: 818-21.

7 Rock WJ, Drance SM, Morgan RW. A modification of the Armaly visual field screening technique for glaucoma. Can J Ophthalmol 1971; 6: 288-92.

8 Hitchings RA, Spaeth GL. The optic disc in glaucoma. I. classification. Br J Ophthalmol 1976; 58: 863-76.

9 Hitchings RA. The optic disc in glaucoma. III. Diffuse optic disc pallor with raised intraocular pressure. Br J Ophthalmol 1978; 62: 670-5.

10 Read, RW, Spaeth GL. A practical and clinical appraisal of the optic disc in glaucoma. Trans Am Acad Ophthalmol Otolaryngol 1974; 22: 225-74.

11 Herschler J, Osler RH. Baring of the circumlinear vessel: an early sign of optic nerve damage. Arch Ophthalmol 1980; 98: 865-9.

12 Osler RH, Herschler J. The significance of baring of the circumlinear vessel. Arch Ophthalmol 1981; 99: 817-8. 